\title{
Effect of Repetitive Transmitter Signals on SQUID Response in Geophysical TEM
}

\author{
Grigory Panaitov, Marcel Bick, Yi Zhang, and Hans-Joachim Krause
}

\begin{abstract}
Results of SQUID application in geophysical time domain transient electromagnetic (TEM) measurements are presented. We analyze peculiarities of SQUID TEM recordings, comparing them to data of commonly used induction coil. Two significant effects have been observed with the SQUID TEM system which are less pronounced or not observed in corresponding reference coil data. We consider a model for SQUID TEM measurements, taking into account the effect of repetitive transmitter signals which gives a possible explanation for these effects.
\end{abstract}

Index Terms- Geophysical measurements, SQUID

\section{INTRODUCTION}

$\mathrm{D}$ UE to an excellent magnetic field sensitivity, the SQUID magnetometer is a very attractive sensor for geophysical applications. Especially in the time domain transient electromagnetic (TEM) exploration, the magnetometer has significant advantages over the commonly used induction coil [1]-[3]. Since 1991, a few HTS SQUID magnetometer systems for ground-based and airborne geophysical TEM exploration have been developed in Australia, Germany and China [2], [4]-[6]. The experimental setup was based on the commonly used TEM technique, with a SQUID magnetometer as a receiver, instead of an induction coil. In numerous field trials, the reliability of the SQUID TEM technique and the advantage of the SQUID magnetometer over the induction coil were clearly demonstrated [2],[4],[6]. However, two significant peculiarities were found in SQUID TEM data, which have not been observed in corresponding reference coil data - the 'frequency dependence' effect of the ground response and the zero-crossover effect of SQUID recorded data [8]. In Ref. [8], we described these two effects based on the results of TEM trials with HTS SQUID magnetometers and proposed a model of repetitive transmitter pulses for their possible explanation. Asten showed earlier [7] that the effect of the full transmitter waveform is very significant in the case of coil recordings above a ground structure with a resistive layer over a conductive basement. We demonstrated in [8] that this effect may be considerable even above a nearly homogeneous ground in the case of SQUID magnetometer receivers. Moreover, the slowly decaying magnetometer transient of a specific layered structure may invert the data sign due to the effect of full

Manuscript received September 17, 2000.

G.Panaitov (E-mail: g.panaitov@fz-juelich.de, phone +49-2461-613231), M.Bick, Y.Zhang, and H.-J.Krause are with the Institute for Thin Film and Ion Technology, Research Center Jülich, D-52425 Jülich, Germany. transmitter waveform. Simulations of TEM transients in [8] were performed taking into account only two previously induced earth responses.

The topic of the present paper is to consider the effect of many remnant responses on TEM recordings. We also present the results of additional laboratory tests to prove the correct operation of the SQUID system under strongly varying primary fields.

\section{SQUID TEST AND TEM MEASUREMENTS}

A HTS SQUID magnetometer with a white noise level of $40 \mathrm{fT} / \mathrm{VHz}$ was used in TEM trials [2]. To generate the electromagnetic field response, a common induction coil ground-based TEM equipment was applied. A square wave primary field was produced by a $100 \times 100 \mathrm{~m}^{2}$ transmitter loop (Fig. 1). The secondary field, i.e., the magnetic field of currents induced in the ground, was recorded by the SQUID magnetometer in the 'in-loop' configuration during the current-off periods between the positive and negative primary field pulses.

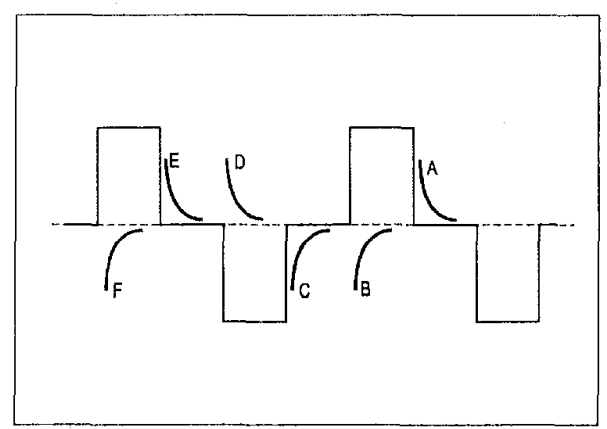

Fig. 1 Secondary field TEM traces A, B, .. F (thick lines) induced in the ground by square wave transmitter signal (thin line).

To improve the signal-to-noise ratio, an averaging of the repetitive secondary fields was performed using standard data acquisition units such as PROTEM or SIROTEM. All magnetometer recordings were compared with induction coil measurements performed as a reference. Generally, a good agreement was demonstrated between SQUID and coil data. However, in numerous SQUID TEM field trials, we observed two significant effects with the SQUID TEM system which were not observed in coil measurements conducted at the same location. At different measuring locations, these effects were more or less pronounced, depending on the ground structure and the conductivity parameters.

The first peculiarity is a 'frequency dependence' effect - 
the slope of the SQUID response depends on the frequency of the transmitter wave signal, decaying more rapidly for higher frequencies. The effect is less pronounced or not visible at all in coil measurements. We did observe this 'frequency dependence' effect at every geophysical location we measured with a SQUID, and it was always reproducible.

The second type of SQUID data peculiarity, a 'zero crossover effect', was observed only above specific ground structures. The recorded data are positive at early times, then go through zero to negative values at late times. This effect of data sign inversion was not observed in corresponding coil recordings. In SQUID magnetometer recordings, however, the data crossover was very reproducible. It was confirmed also by another German group [9] using a dc-SQUID. In [4], Foley et al. report about a sign reversal which is possibly similar to our observations, but the reason for that effect was not identified.

In principle, the observed sign reversal transient could be a 'false' anomaly, caused by drift in the SQUID electronics or flux relaxation effects in the SQUIDs. In order to exclude a possible 'instrumental' origin of these effects, additional laboratory tests of our SQUID TEM system were performed. During the TEM measurements, the SQUID is exposed to the abruptly varying square wave primary field of about $100 \mathrm{nT}$. In principle, the variation of a strong magnetic field could produce an unwanted magnetic flux movement in the HTS film of the SQUID, thus disturbing the TEM recordings. We tested our SQUID system in magnetic shielding, applying a magnetic field with a time profile similar to that of a typical TEM primary field. The field was generated by a small induction coil. Care was taken not to place electrically conducting material close to the setup, thus no secondary fields were induced. The recorded field decay was compared with the shape of the turn-off ramp of the primary field. Figure 2 shows SQUID recordings taken with transmitter frequencies $1.25 \mathrm{~Hz}, 5 \mathrm{~Hz}$ and $20 \mathrm{~Hz}$, for two different cases of exponential excitation decay, with time constants of $\tau=0.12 \mathrm{~ms}$ (squares) and $\tau=1.2 \mathrm{~ms}$ (triangles), respectively. Solid circles denote the SQUID noise, measured in absence of any primary

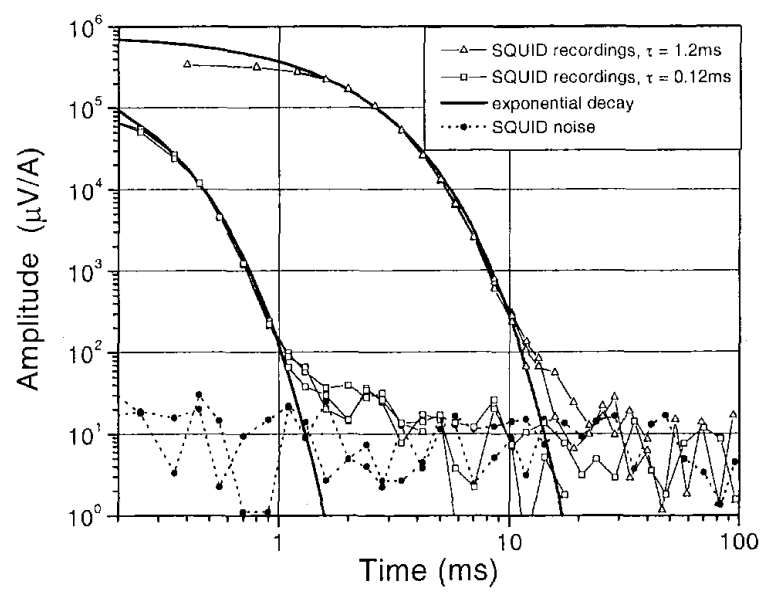

Fig. 2, SQUID recordings (open symbols) in exponentially decaying primary fields with time constants of $0.12 \mathrm{~ms}$ and $1.2 \mathrm{~ms}$ (thick lines), compared to the SQUID noise without primary field (solid symbols). field. It is obvious that there are no parasitic effects and no noise increase after the decay of the transmitter pulse. The SQUID signal decay approaches an exponential (thick lines), with time constants defined by the RC lines in the transmitter coil. Results of these measurements and additional tests of our SQUID electronics show that an instrumental character of the above TEM peculiarities is very unlikely in our case.

In [8], we explain the phenomena described above as a consequence of the repetitive transmitter waveform. This effect has previously been shown to be of significance in the case of coil measurements over a conducting basement with a resistive upper layer [7], [11]. However, when using a magnetometer sensor such as a SQUID in TEM, the effect of repetitive transmitter signals may be considerable even over a nearly homogeneous ground [8]. At some specific structures like a layered ground with a very slow decay, magnetometer responses may even undergo a sign inversion at late times. Both these effects are generally not visible in coil recordings, due to much faster response decay. In [8], we analyze both effects by simulating the transients taking into account two previously induced remnant responses.

In the following section, we discuss the effect of a repetitive transmitter waveform in more detail. We present simulations of the SQUID and coil recordings, taking into account up to 11 remnant responses.

\section{MODELING ANALysis OF TEM DATA}

As mentioned above, we used a square waveform transmitter signal (Fig. 1) which induces repetitive eddy currents in the ground. Turn-off of the positive primary field induces eddy currents corresponding to a positive secondary field response (traces $\mathrm{A}$ and $\mathrm{E}$ in Fig. 1), while a negative primary field turn-off induces a negative field response (trace $\mathrm{C}$ in Fig. 1). In order to improve the signal-to-noise ratio, $\mathrm{N}$ positive and negative transients, $\mathrm{V}(\mathrm{t})$, are averaged in the data acquisition unit, as

$$
\bar{V}(\mathrm{t})=\frac{1}{2 \mathrm{~N}} \sum_{n=1}^{N}\left[\mathrm{~V}_{n}^{+}(\mathrm{t})+\mathrm{V}_{\mathrm{n}}^{-}(\mathrm{t})\right]
$$

A second series of positive and negative field responses are induced by negative and positive primary field turn-ons (traces D and B, respectively). Usually, it is assumed that during the recording of an actual positive response (e.g., $\mathrm{A}$ in Fig. 1), the negative current ring associated with the previous negative field step (B) has already decayed to zero or is negligibly small. Generally, this holds true for a homogeneous ground with a rapidly decaying coil response: $\mathrm{V}_{c}(\mathrm{t}) \sim \mathrm{dB} / \mathrm{dt} \sim$ $\sigma^{3 / 2} t^{(-5 / 2)}$, where $\sigma$ is the ground conductivity [10]. But the SQUID response decays much slower than the coil transient: $\mathrm{V}_{\mathrm{s}}(\mathrm{t}) \sim \mathrm{B}(\mathrm{t}) \sim \sigma^{3 / 2} \mathrm{t}^{(-3 / 2)}$. Moreover, in multi-layered structures, e.g., in the case of a two-layer ground with an upper resistive layer overlying a well-conducting basement, a transient decay may be much slower than in the case of a homogeneous ground [10]. Many non-negligible current rings may persist simultaneously in the ground. This will disturb the recording of the original ground response. In the case of a magnetometer sensor measuring the B-field directly, this 
disturbance will be more significant than in the case of an induction coil sensor which measures the more rapidly decaying time derivative $\mathrm{dB} / \mathrm{dt}$. In the following, all remnant responses ( $B, C, D \ldots$, in Fig. 1) will be referred to as 'disturbing' responses. The averaged transient signal, taking into account all the disturbing remnant responses, may be expressed as follows:

$$
\overline{\mathrm{V}}(\mathrm{t})=\frac{1}{2 \mathrm{~N}} \sum_{n=\mathrm{i}}^{N}\left[\begin{array}{l}
\sum_{k=0}^{2 n-1}(-1)[\mathrm{V}(\mathrm{t}+2 k \mathrm{~T})-\mathrm{V}(\mathrm{t}+2 k \mathrm{~T}+\mathrm{T})]+ \\
+\sum_{k=0}^{2 n-2}(-1)[\mathrm{V}(\mathrm{t}+2 k \mathrm{~T})-\mathrm{V}(\mathrm{t}+2 k \mathrm{~T}+\mathrm{T})]
\end{array}\right]
$$

where $\mathrm{T}=1 / 4 \mathrm{f}$ denotes the time delay between neighboring responses. Thus, according to (2), the recorded field decay will depend on the transmitter frequency, $f$. To evaluate how significant this dependence may be, we performed a numerical calculation of (2) in the case of a ground response of the form $V(t)=\alpha t^{-\beta}$. In the whole range $1<\beta<\infty$, the sum (2) is converging to finite values, even for an infinite number of pulses, $\mathrm{N} \rightarrow \infty$. In TEM exploration, $\mathrm{N}$ typically ranges between 100 and few thousands. Obviously, most of the remnant responses are negligibly small. To simulate the SQUID TEM data of a nearly homogeneous ground shown in Fig. 3, we used the basic response function $V(t)=\alpha t^{-\beta}$, with fit parameters $\alpha=3000$ and $\beta=1.2$. Fig. 3 presents a simulation of SQUID data for $f=25,5$, and $1.25 \mathrm{~Hz}$, taking into account 11 disturbing responses (thick lines). The simulation fits very well to the recorded SQUID transients (circles). A simulation with only 1 remnant response (dotted line) gives almost the same result. This indicates that here only the first remnant response contributes significantly to the SQUID recording. For the considered ground parameters $\alpha=3000$ and $\beta=1.2$, all terms $V(t+2 k T)$ with $k \gg 1$ in (2) are negligible. In order to simulate the coil response, the time derivative of the SQUID simulation is evaluated. One can see that the deviation

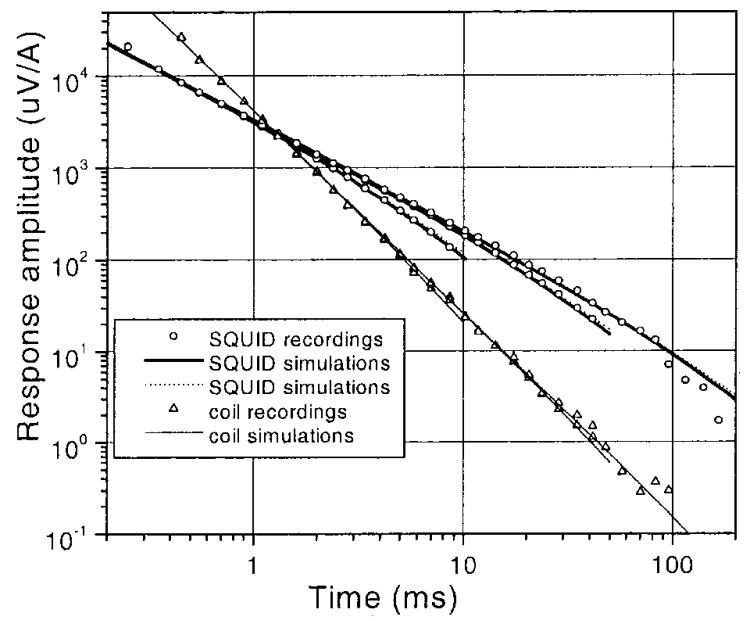

Fig. 3. Simulation of SQUID (thick lines) and coil (thin lines) transients taking into account 11 remnant responses for 3 transmitter frequencies: 25 , 5 , and $1.25 \mathrm{~Hz}$. Circles and triangle symbols are the corresponding SQUID and coil recordings. Dotted lines are simulations of SQUID transients taking into account only 1 remnant response. between coil simulations (thin lines) is smaller than the scattering of coil data (triangles). Therefore, the frequency dependence effect could not be observed in these coil recordings. This is because the coil signal decays faster than the SQUID signal and the disturbing secondary fields become negligible small compared to the actually recorded field response.

A further analysis of (2) shows that the influence of remnant disturbing responses may result in effects even stronger than a simple frequency dependence. Although the calculations show that (2) is always positive for homogeneous ground responses like $V(t)=\alpha t^{-\beta}$, the expression (2) can be even negative for a multi-layered ground with a more complicated response $\mathrm{V}(\mathrm{t})$. We illustrate this for the example of a two-layered ground. According to [10], the response decay of a two-layered ground with an upper resistive layer schematically consists of three parts. A fast transient decay at early time $\left(t<t_{1}\right)$ is followed by a very slow decay in intermediate time $\left(t_{1}<t<t_{2}\right)$ and then, at $t>t_{2}$, the response signal drops quickly down. It is obvious that remnant responses at $t>t_{2}$ are negligibly small. Only few slowly decaying responses which didn't reach this late time interval (i.e., $t<t_{2}$ ) will give a significant contribution to the recorded response. Fig. 4 shows an example of a SQUID response simulation taking into account two remnant disturbing responses. The simulation is based on the two slope response $V(t)=\alpha_{1} t^{-\beta 1}+\alpha_{2} t^{-\beta 2}$ of a two-layered ground. Parameters $\alpha_{1}=1$, $\alpha_{2}=500, \beta_{1}=5.5$, and $\beta_{2}=0.44$ were chosen to fit to the measured data. For $\mathrm{T}=50 \mathrm{~ms}$, which was also used in the measurements, the simulation (solid line) correlates well with the SQUID data (symbols). Both simulation and measurement show a zero-crossover at $t=18 \mathrm{~ms}$. The negative data branch is inverted to plot the whole transient in the double logarithmic scale. The simulation of the coil transient (thick line in Fig. 5) also correlates well with the experimental coil recordings (squares) which show no sign inversion.

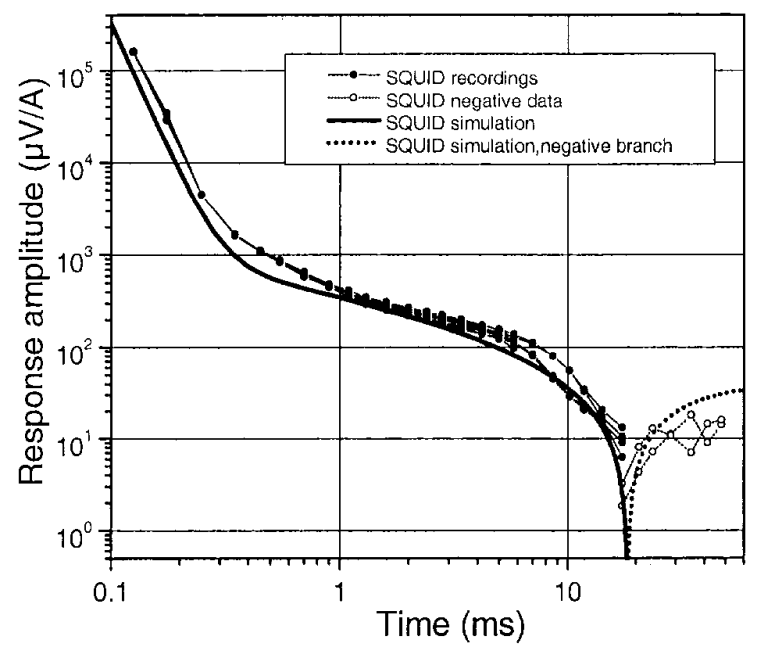

Fig. 4. Simulation of SQUID TEM response at Teveren (Germany). Both synthetic (thick line) and recorded SQUID data (symbols) show the zerocrossover at about $18 \mathrm{~ms}$. Late time data (open circles) and simulation (dotted line) are inverted to plot in double-logarithmic scale. 


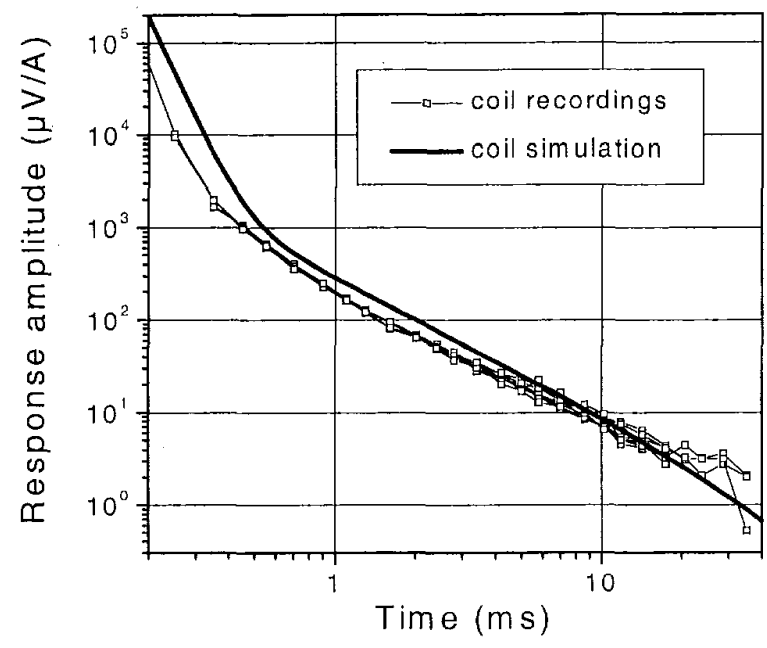

Fig. 5. Simulation of coil (thick line) TEM data at Teveren. Symbols are coil recordings. Both synthetic and recorded data show no zero-crossover.

An important issue in the full transmitter waveform consideration is the number of significant remnant responses. For a simple one slope ground response like in Fig. 2, the model holds true for any $\mathrm{k} \gg>1$ in (2). As an example, we showed that simulations with 11 and 1 remnant responses are both in good agreement with the recorded data, showing the 'frequency dependence' effect (Fig. 3). In this case, only the first disturbing response dominates the effect. The contribution of the higher-order responses is negligible. In the case of a complex multi-layered ground, the situation is quite different. Here, the number of remnant responses, $n$, which are taken into account, is crucial. The precise calculation of the effect is possible taking into account all terms in (2). In practice, however, this is problematic because evaluation of the expression (2) requires knowledge of the response transient $V(t)$ even at very late times, $t>T$. For a complex multi-layered ground, it is very difficult to predict the

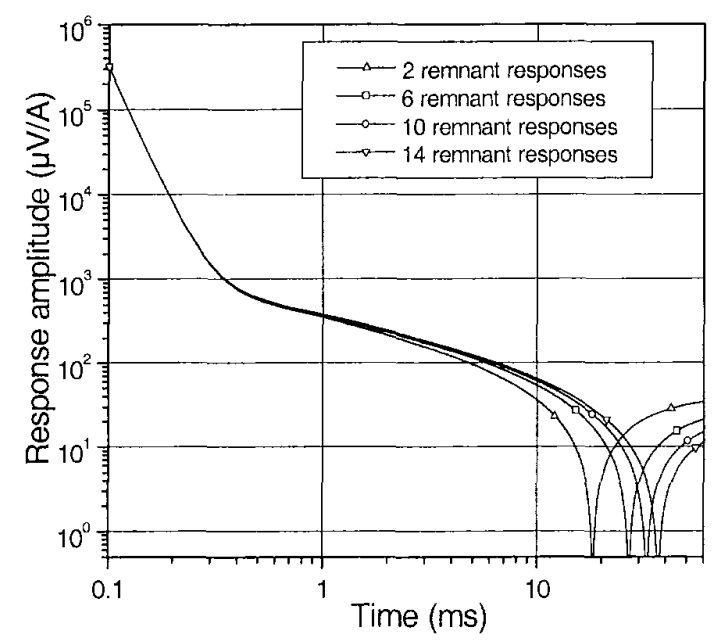

Fig. 6. Simulation of the SQUID TEM response tacking into account 2, 6, 10 and 14 remnant responses. Two slope response of a two-layered ground was used as a basic simulation function: $V(t)=t^{-5.5}+500 t^{-0.44}$. transient behavior at very late times. Therefore, in our simulations we take into account only a limited number of remnant responses. Depending on the number $\mathrm{n}$ the series is truncated at, the result of the simulation may dramatically change. Fig. 6 shows this dependence in the case of the synthetic transient from Fig. 4, for $n=2,6,10$ and 14. The time of the zero-crossover shifts considerably. Therefore, the simulation of the zero-crossover effect in Fig. 4 should be considered only as an example, demonstrating the possibility of data inversion due to the effect of remnant disturbing responses.

In summary, we analyzed the effect of a repetitive transmitter signal on SQUID TEM. Our simulations show that in the case of a nearly homogeneous ground, the effect of the full waveform leads to a frequency dependence of the magnetometer response. Often, only the first few disturbing remnant responses are significant. For a complex multilayered ground, the number of significant remnant responses may be crucial. In some cases, the repetitive waveform may result in a sign inversion of the magnetometer response. These effects are much less pronounced or not observable at all in induction coil recordings.

\section{ACKNOWLEDGMENT}

We would like to thank A. Hördt, S. Helwig and A. I. Braginski for fruitful discussions.

\section{REFERENCES}

[1] C. P. Foley and K. E. Leslie, "Potential use of high Tc SQUIDs for airborne electromagnetics," Exploration Geophysics, vol. 29, pp. 3034, 1998.

[2] M. Bick, G. Panaitov, Y. Zhang, H. Bousack, A. I. Braginski, U. Kalberkamp, H. Burckhardt, and U. Matzander, "A HTS rf SQUID vector magnetometer for geophysical exploration methods," IEEE Trans. Appl. Supercond., vol. 9, No. 2, pp. 3780-3785, 1999.

[3] B. R. Spies, "Depth of investigation in electromagnetic sounding methods," Geophysics, vol. 54, pp. 872-888, 1989.

[4] C. P. Foley et al. "Field trials using HTS SQUID magnetometers for ground-based and airborne gcophysical applications," IEEE Trans. Appl. Supercond, vol. 9, No. 2, pp. 3786-3792, 1999.

[5] S. G. Wang, L. H. Zhang, C. I. Wang, Y. D.Dai, "Application of HTS SQUID devices in transient electromagnetic method for magnetotelluric soundings," Physica C, vol. 282-287, pp. 411-414, 1997.

[6] U. Kalberkamp, U. Matzander, K.-D. Huscmann, G. Panaitov, E. Zimmermann, Y. Zhang, "HTS rf-SQUID applications to geophysical exploration methods," Applied Superconductivity, vol. 5, issue 7-12, pp. 205-211, 1999.

[7] M. Asten, "Full transmitter waveform transient electromagnetic modelling and inversion for soundings over coal measures," Geophysics, vol. 52, No.3, pp.279-288, 1987.

[8] G. Panaitov, M. Bick and Y. Zhang, "Peculiarities of SQUID applications in geophysical TEM," submitted for publication in Geophysics, 2000 .

[9] A. Chwala, R. Stolz, J. Ramos, V. Schultze, H.-G. Meyer and D. Kretzschmar. "An HTS dc-SQUID system for geomagnetic prospection," Supercond. Sci. Technol., vol. 12, pp. 1036-1038, 1999.

[10] B. R. Spies and F. C. Frischknecht, "Electromagnetic sounding," in Electromagnetic methods in applied geophysics, M. N. Nabighian, Ed. Oklahoma: Society of Exploration Geophysicists, 1991.

[11] B. R. Spies, "An investigation of energy storage technology for deep electromagnetic sounding", J. Geomag. Geoelectr, vol. 45, pp. 917$930,1993$. 